\title{
A new strategy for prevention of anastomotic stricture using tacrolimus-eluting biodegradable nanofiber
}

\author{
Masato Mutsuga, MD, ${ }^{a}$ Yuji Narita, MD, PhD, ${ }^{\text {a,b }}$ Aika Yamawaki, BMedEng, ${ }^{\text {b }}$ Makoto Satake, PhD, ${ }^{\mathrm{c}}$ Hiroaki Kaneko, PhD, \\ Yoshihiro Suematsu, MD, PhD, ${ }^{\mathrm{d}}$ Akihiko Usui, MD, PhD, ${ }^{\mathrm{a}}$ and Yuichi Ueda, MD, $\mathrm{PhD}^{\mathrm{a}}$
}

\begin{abstract}
Objective: We developed a novel sustained drug-eluting device using tacrolimus-eluting biodegradable nanofiber to prevent anastomotic stricture and evaluated the effects in a rat abdominal aortic anastomosis model.
\end{abstract}

\begin{abstract}
Methods: In vitro and in vivo tacrolimus release tests for tacrolimus-eluting biodegradable nanofiber were performed to confirm its sustained release. To verify the prevention of anastomotic stricture, tacrolimus-eluting biodegradable nanofiber was placed around the end-to-end anastomosis of abdominal aorta in rats. Five rats were allocated to the following 5 groups: (1) control without tacrolimus-eluting biodegradable nanofiber, (2) $5 \mathrm{mg}$ of nanofiber only ( $0 \mathrm{wt} \%$ of tacrolimus), (3) $5 \mathrm{mg}$ of tacrolimus-eluting biodegradable nanofiber containing $0.04 \mathrm{wt} \%$ of tacrolimus, (4) $5 \mathrm{mg}$ of tacrolimus-eluting biodegradable nanofiber containing $0.1 \mathrm{wt} \%$ of tacrolimus, and (5) $5 \mathrm{mg}$ of tacrolimus-eluting biodegradable nanofiber containing $1.0 \mathrm{wt} \%$ of tacrolimus. Morphometric and histologic analyses including immunohistochemistry were performed in each of the groups 2 weeks after the operation.

Results: The tacrolimus-eluting biodegradable nanofiber gradually released tacrolimus for at least 1 month in
vitro and in vivo. The ratio of intimal area was significantly reduced in the $1.0 \mathrm{wt} \%$ tacrolimus-eluting biode-
gradable nanofiber group compared with the other groups $(0.26,0.24,0.25,0.21$, and 0.08 in control, $0 \mathrm{wt} \%$,
$0.04 \mathrm{wt} \%, 0.1 \mathrm{wt} \%$, and $1.0 \mathrm{wt} \%$, respectively, $P<.05)$. The cells, which constitute intimal hyperplasia, were
positive for smooth muscle actin and SMemb, and factor VIII revealed that endothelial cells covered the surface
of the aortic lumen even in the $1.0 \mathrm{wt} \%$ tacrolimus-eluting biodegradable nanofiber group in immunohisto-
chemistry.
\end{abstract}

Conclusion: Tacrolimus-eluting biodegradable nanofiber reduced neointimal hyperplasia and preserved endothelialization. This device may be useful in the prevention of anastomotic stricture.

Anastomotic stricture has remained the main reason for arterial, venous, arteriovenous, and prosthetic graft failure. ${ }^{1-5}$ In the area of coronary surgery, the surgical outcome of coronary artery bypass grafting has remarkably improved, but although arterial grafts have enhanced the long-term patency rate, the long-term patency of saphenous vein grafts has been limited. ${ }^{1,2}$ Approximately $20 \%$ of arterial grafts still show evidence of occlusion or stenosis in the late period. ${ }^{3}$ The surgical outcome of an arteriovenous shunt has also been limited, and its failure has caused severe morbidity. ${ }^{4}$ The prosthetic grafts used for peripheral bypass and hemodialysis access have shown poor patency during the past few

\footnotetext{
From the Nagoya University, Graduate School of Medicine, Department of Cardiotho-

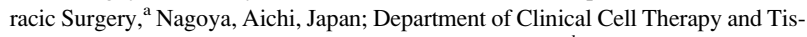
sue Engineering, Nagoya University School of Medicine, ${ }^{\mathrm{b}}$ Nagoya, Aichi, Japan; Department of Tissue Engineering Development, Teijin Technology Innovation Center, Teijin Limited, ${ }^{\mathrm{c}}$ Hino, Tokyo, Japan; and the Department of Cardiac Surgery, The University of Tokyo Graduate School of Medicine, ${ }^{\mathrm{d}}$ Tokyo, Japan.

Received for publication June 19, 2008; revisions received Oct 2, 2008; accepted for publication Nov 15, 2008.

Address for reprints: Yuji Narita, MD, PhD, Department of Clinical Cell Therapy and Tissue Engineering, Nagoya University School of Medicine, 65 Tsurumaicho, Syowa-ku, Nagoya, Aichi 466-8550, Japan (E-mail: ynarita@med.nagoya-u.ac.jp). J Thorac Cardiovasc Surg 2009;137:703-9

$0022-5223 / \$ 36.00$

Copyright (c) 2009 by The American Association for Thoracic Surgery doi:10.1016/j.jtcvs.2008.11.017
}

years. ${ }^{5}$ In addition to technical failure, the anastomotic stricture, whether the anastomosis is end to end or end to side, is largely affected by the degree of neointimal hyperplasia, which develops immediately after graft anastomosis. ${ }^{6}$ Preventing anastomotic stricture is the key to long-term efficacy of all types of vascular anastomosis and improves the morbidity and prognosis of patients.

Percutaneous coronary intervention with drug-eluting stents, which was introduced in 2003, has dramatically improved restenosis rates compared with bare metal stents because the drug gradually prevents neointimal overgrowth around the stent. ${ }^{7}$ We developed a novel sustained drug-releasing device to prevent anastomotic stricture. Our new device is based on the prevention effect against neointimal overgrowth by sustained release of the drug according to the method of drug-eluting stents. The sustained-releasing system is composed of biodegradable polymer, which dissolves continuously little by little, releasing the drug spontaneously. We chose tacrolimus for the drug to prevent neointimal hyperplasia because it has been evaluated in previous studies as an agent to prevent neointimal hyperplasia with respect to release kinetics, effective dosage, safety in clinical practice, and benefit. However, it is difficult to determine what drug is the best to prevent neointimal hyperplasia. $^{8-10} \mathrm{Gel}$, film, and other types of polymer have been 


\section{Abbreviations and Acronyms \\ $\mathrm{H} \& \mathrm{E}=$ hematoxylin-eosin \\ TEBN $=$ tacrolimus-eluting biodegradable nanofiber \\ VSMC $=$ vascular smooth muscle cell}

reported for a drug-releasing system to prevent neointimal hyperplasia. ${ }^{11-14}$ However, it is not clear which type of system is preferable. These devices may not fit a complicated anastomosis, such as an end-to-side anastomosis, because of the lack of material flexibility.

We developed a nano-scale "cotton-wool" configuration fiber by an electrospinning method to fit any type of anastomosis. Tacrolimus, a field-proven antistricture agent, is mixed with this biodegradable fiber for sustained release to create tacrolimus-eluting biodegradable nanofiber (TEBN). This type of cotton wool is well suited to perivascular application. The aim of this study is to evaluate the effects of TEBN for prevention of anastomotic stricture and to estimate the drugreleasing level in a rat abdominal aortic anastomosis model.

\section{MATERIALS AND METHODS \\ Animals}

The animals were cared for in accordance with the "Guide for the Care and Use of Laboratory Animals" published by the US National Institutes of Health (Publication 85-23, National Academy Press, Washington, DC, revised in 1996). All procedures involving animals were approved by the Animal Experiment Advisory Committee of the Nagoya University School of Medicine. In this study, we used male Wistar rats 8 to 10 weeks old (weighing $300-350 \mathrm{~g}$ ) that were purchased from the Chubu Kagaku Shizai Corporation (Nagoya, Japan).

\section{Fabrication of Tacrolimus-Eluting Biodegradable Nanofiber}

We focused on tacrolimus as an antistricture agent because it has been proved to be effective not only for immunosuppression but also for treatment of scars or stent restenosis. ${ }^{10,15,16}$ Tacrolimus (Fermentel Ltd, Jerusalem, Israel) was mixed with biodegradable polymer to achieve sustained release. Our expectation was that tacrolimus would be released when the biodegradable polymer was dissolved. We prepared poly(L-lactide-co-glycolide), which consists of approximately $50 \%$ poly lactic acid and $50 \%$ poly glycol acid, for the biodegradable polymer. To fabricate the nano-scale "cotton-wool" configuration fiber, an electrospinning procedure was used. The polymer solution was added by using a 1-mL syringe with a right angle-shaped metal capillary attached to it. The circular orifice of the capillary had an inner diameter of $1.2 \mathrm{~mm}$. A flat counter electrode was located $35 \mathrm{~cm}$ from the capillary tip. Pressure was applied to the solution in the syringe, gradually forcing the piston to maintain a steady flow of solution from the capillary outlet. The flow rate of the polymer solution was $0.3 \mathrm{~mL} / \mathrm{min}$. The applied voltage was in the range of 10 to $15 \mathrm{kV}$. The fibers released in the atmosphere were electrostatically removed and trapped with a rod-shaped collector located 20 $\mathrm{cm}$ from the capillary tip. The nanofiber ranged from 100 to $800 \mathrm{~nm}$ in diameter. The shape of the TEBN shows a "cotton wool"-like formation (Figure 1). This configuration is flexible and easy to handle for any type of vascular anastomosis. The maximum tacrolimus content in the TEBN was $1.0 \mathrm{wt} \%$.

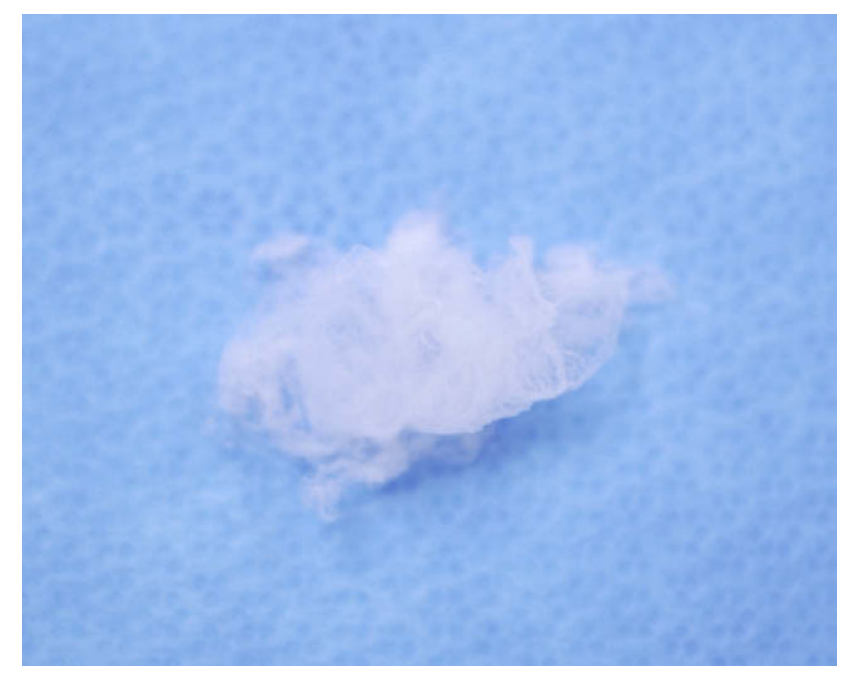

FIGURE 1. Overall appearance of TEBN. TEBN looked like a "cotton wool" formation.

\section{In Vitro Release of Tacrolimus from Tacrolimus- Eluting Biodegradable Nanofiber}

The in vitro release of tacrolimus from the TEBN was evaluated by measurement of the remaining tacrolimus concentration in the TEBN, which was incubated in phosphate-buffered saline ( $\mathrm{pH} 7.4$ ), in the following conditions. Briefly, $10 \mathrm{mg}$ containing $1.0 \mathrm{wt} \%$ of TEBN was incubated in $1 \mathrm{~mL}$ of phosphate-buffered saline at $37^{\circ} \mathrm{C}$, centrifuged, and lyophilized, and the dry weight was measured. TEBN was dissolved in ethyl acetate, and the polymer (poly[L-lactide-co-glycolide]) was removed by ethanol precipitation. The extracts were dissolved in ethyl alcohol, and the concentration of tacrolimus was measured with high-performance liquid chromatography. The concentration of tacrolimus from TEBN was measured at each time point $(1,4,7,14,21$, and 28 days, $\mathrm{n}=3$ at each time point). Measurement data were expressed as a percentage of the volume of tacrolimus originally contained in the TEBN.

\section{In Vivo Release of Tacrolimus from Tacrolimus- Eluting Biodegradable Nanofiber and Side Effects of Tacrolimus-Eluting Biodegradable Nanofiber}

The in vivo release of tacrolimus from TEBN was also measured. Five milligrams of TEBN containing $1.0 \mathrm{wt} \%$ of tacrolimus was subcutaneously implanted into the backs of 30 Wistar rats. Five rats each were sacrificed 1 , $3,5,7,14$, and 28 days after implantation. The remaining TEBN in the back was collected, and the tacrolimus concentration in the TEBN was measured in the same manner described above.

To verify the side effects of TEBN in the implanted rats, the blood concentration of tacrolimus (enzyme-linked immunosorbent assay; SRL, Inc, Tokyo, Japan) was measured. This kit is useful for the therapeutic drug monitoring of tacrolimus in the subtherapeutic and low therapeutic ranges. Complete blood count, liver function (serum concentration of glutamic oxaloacetic transaminase, glutamic pyruvate transaminase, and gamma-guanosine triphosphate), renal function (serum concentration of blood urea nitrogen and creatinine), and pancreatic enzyme (amylase) tests were also performed in these rats at each time point, and the results were compared with those of sham-operated rats.

\section{Operative Procedures and Treated Groups}

The experiments were carried out on 25 Wistar rats. General anesthesia was induced with etherization and maintained with 25 to $50 \mathrm{mg} / \mathrm{kg}$ of 


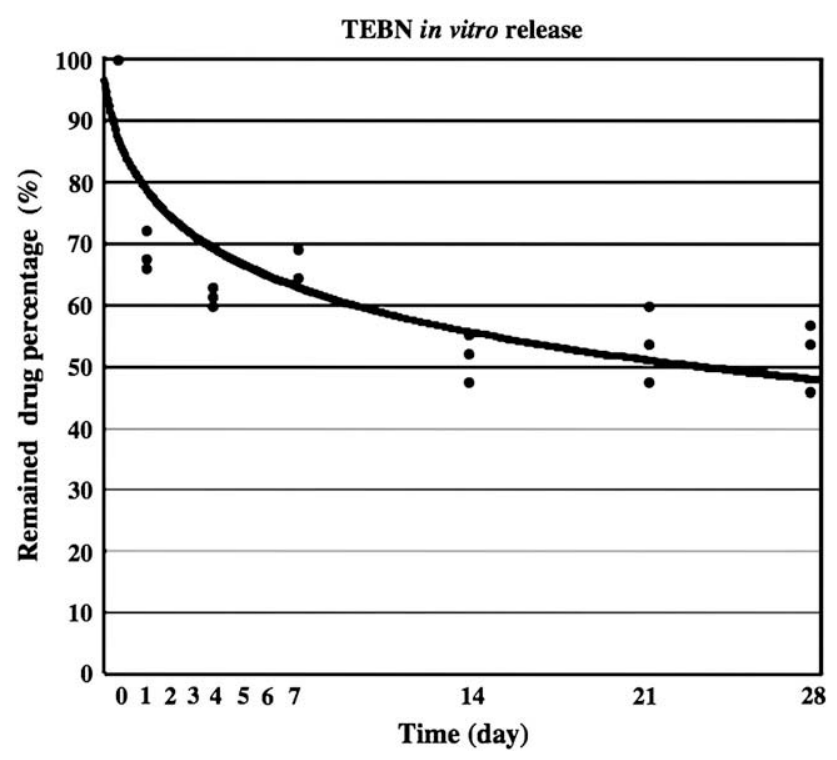

FIGURE 2. In vitro tacrolimus-releasing test with TEBN. Longitudinal axis of this graph indicates the remaining percentage of tacrolimus in the TEBN (\%). The remaining tacrolimus curve seems to be logarithmic. $T E B N$, Tacrolimus-eluting biodegradable nanofiber.

pentobarbital sodium (Kyoritsu Seiyaku Corp, Tokyo, Japan) and $0.01 \mathrm{mg} /$ $\mathrm{kg}$ of atropine sulfate (Mitsubishi Tanabe Pharma Corp, Osaka, Japan) injected into the peritoneal cavity. An abdominal midline incision was made to enter the peritoneal cavity, and the infrarenal abdominal aorta was simply clamped and divided. The end-to-end anastomosis was made by a continuous running suture with 9-0 silk (NESCO SUTURE, Alfresa Pharma Corp, Tokyo, Japan). Neither anticoagulant nor antiplatelet agents were administered throughout the procedure.

Rats were randomly divided into 5 groups ( $\mathrm{n}=5$ in each group): (1) control without TEBN, (2) $5 \mathrm{mg}$ of nanofiber (without tacrolimus), (3) $5 \mathrm{mg}$ of TEBN containing $0.04 \mathrm{wt} \%$ tacrolimus, (4) $5 \mathrm{mg}$ of TEBN containing 0.1 $\mathrm{wt} \%$ tacrolimus, and (5) $5 \mathrm{mg}$ of TEBN containing $1.0 \mathrm{wt} \%$ tacrolimus. Rats were sacrificed 2 weeks after operation and evaluated with the following procedures.

\section{Histologic Analysis and Ratio of Intima/Vessel wall (Intima + Media) Area}

For the histologic analysis, the anastomotic sites were resected beyond the suture lines, fixed with $7.5 \%$ buffered formaldehyde (Sigma Aldrich Japan, Tokyo, Japan), and embedded in paraffin. Short axial consecutive 5$\mathrm{mm}$ serial sections were obtained just around the suture anastomosis. Three individual and random sections from the anastomosis site were evaluated. The sections were stained with hematoxylin-eosin $(\mathrm{H} \& \mathrm{E})$ for morphometric analysis. The area of the intima (including neointima) and media at the anastomosis site was measured on the sections stained with H\&E. To achieve a reproducible result, the mean value of these areas (intima and media) from 3 sections was adopted. To characterize neointimal hyperplasia, the ratio of intimal area was calculated with the following formula: ratio of intimal area $=$ intimal area/ (intimal + median area). This morphometric analysis was performed using Image $\mathrm{J}$ for Mac OS X (Wane Rasband, National Institutes of Health, Bethesda, Md).

\section{Immunohistochemical Analysis for Neointimal Hyperplasia and Endothelial Cells}

Immunohistochemical staining was performed to identify the cell, which comprised the intimal hyperplasia, and to evaluate reendothelialization at

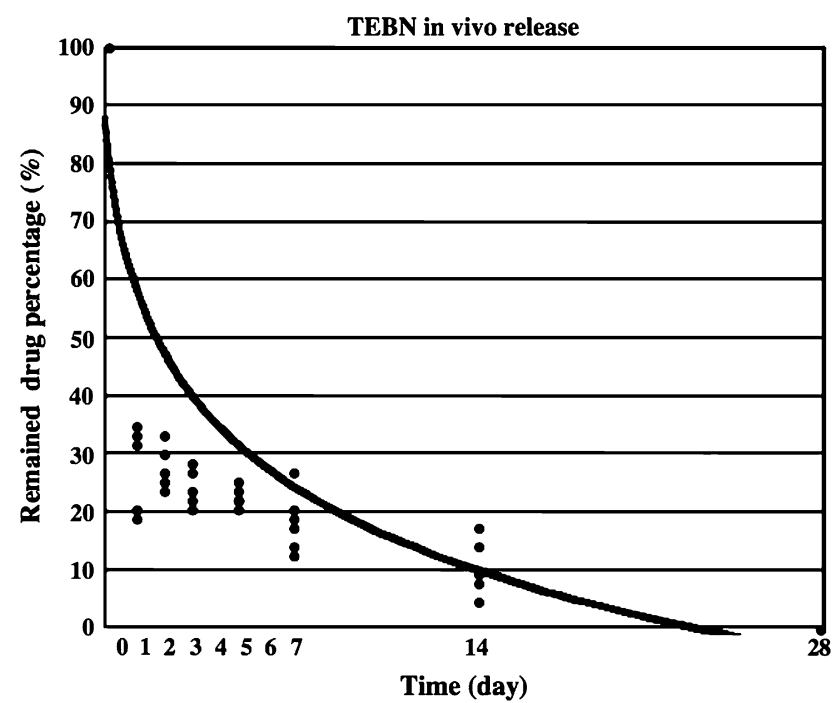

FIGURE 3. In vivo tacrolimus-releasing test with TEBN. Longitudinal axis of this graph indicates remaining percentage of tacrolimus in the TEBN (\%). Tacrolimus was released at a fast initial burst phase during the first few days followed by a slow sustained phase during the following 4 weeks. TEBN, Tacrolimus-eluting biodegradable nanofiber.

the injured anastomotic site. To label mesenchymal cells (eg, smooth muscle cell or myofibroblast) and endothelial cells, alpha-smooth muscle actin antibody (1:3000) (Sigma-Aldrich Inc., St. Louis, Mo), SMemb (1:3000) (Yamasa Co., Tokyo, Japan), and factor VIII antibody (ready-to-use for immunohistochemical staining) (NeoMarker, Fremont, Calif) were used as primary antibodies. Biotinylated secondary antibody (1:400) (anti-mouse immunoglobulin-G; Vector Laboratories, Burlingame, Calif) was applied, and avidin-biotin complex Vecstain ABC kit (Vector Laboratories, Burlingame, Calif) was used for visualization. Slides were developed in diaminobenzidine solution and counterstained with $\mathrm{H} \& \mathrm{E}$.

\section{Statistical Analysis}

Statistical analysis was performed using Excel for Mac with the add-in software Statview for Mac (Version 5.0, SAS Institute Inc, Cary, N.C.). Data were analyzed by 1-way analysis of variance, and multiple comparisons were made by the Scheffe procedure. Experimental results were expressed as mean \pm standard deviation.

\section{RESULTS}

All animals survived to the end of our protocol, and no sign of infection was detected.

\section{Tacrolimus Release from Tacrolimus-Eluting Biodegradable Nanofiber}

In vitro release of tacrolimus from TEBN is shown in Figure 2. Approximately $30 \%$ of tacrolimus was released into the solution from TEBN on the first day, but release persisted thereafter, showing $48 \%$ release at 2 weeks and $49 \%$ release at 4 weeks after incubating TEBN in phosphate-buffered saline.

In vivo release profiles of tacrolimus from TEBN are shown in Figure 3. Early burst release was also observed in vivo. Approximately $70 \%$ of the tacrolimus was released from TEBN within the first day. After the initial burst phase 


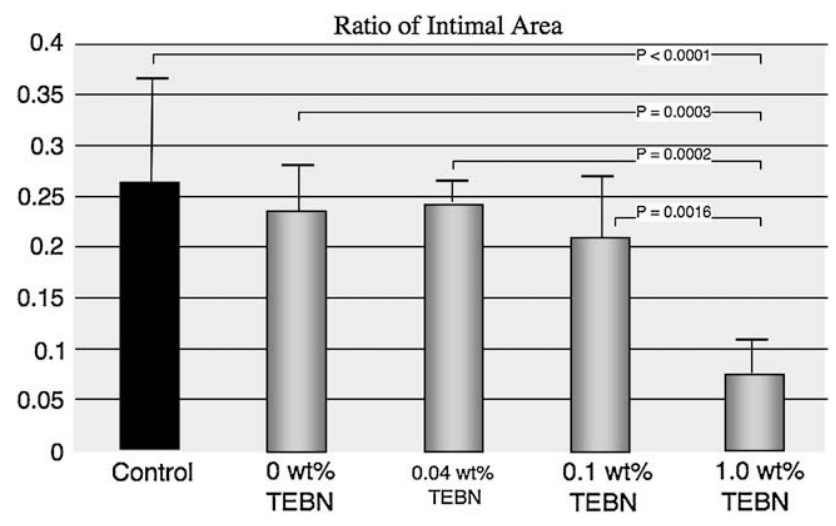

FIGURE 4. Ratio of intimal area. The neointimal hyperplasia is significantly inhibited by $1.0 \mathrm{wt} \%$ TEBN. TEBN, Tacrolimus-eluting biodegradable nanofiber.

during the first few days, a slow release phase followed for 4 weeks. The in vivo experimental study confirmed the gradual sustained release of tacrolimus from TEBN.

\section{Ratio of Neointimal-Hyperplasia and Immunohistochemical Staining}

The ratio of intimal area (intimal area divided by the intimal and medial area) in the control without TEBN, $5 \mathrm{mg}$ of nanofiber only, $5 \mathrm{mg}$ of TEBN, and containing $0.04 \mathrm{wt} \%$, $0.1 \mathrm{wt} \%$, and $1.0 \mathrm{wt} \%$ tacrolimus were $0.26,0.24,0.25$, 0.21 , and 0.08 , respectively (Figure 4). Only TEBN with
$1.0 \mathrm{wt} \%$ of tacrolimus inhibited neointimal hyperplasia significantly. Anastomotic sections stained with H\&E are shown in Figure 5. The cells constituting the intimal hyperplasia were alpha-smooth muscle actin antibody and SMemb positive in immunohistochemistry. Endothelial cells, which were stained with factor VIII immunohistochemically, covered the surface of the aortic lumen even in the $1.0 \mathrm{wt} \%$ TEBN group, in which neointimal hyperplasia was significantly inhibited (Figure 6).

\section{Consideration of Side Effects}

There was no difference between the TEBN group and the sham group in complete blood count, liver function test results, renal function test results, and pancreatic enzymes. There was no blood concentration of tacrolimus in either group. There was no anastomotic aneurysm or pseudoaneurysm formation during the 2 weeks of the protocol.

\section{DISCUSSION}

The main finding of this study was that gradual sustained release of tacrolimus from TEBN applied perivascularly inhibited neointimal hyperplasia and preserved endothelialization after anastomotic stricture. Tacrolimus, a broadspectrum immunosuppressant drug discovered in 1984, inhibits the progression of the cell cycle from the G1 to the $\mathrm{S}$ phase of the mitotic cycle in the same manner as sirolimus. It has been widely used for reducing the incidence and
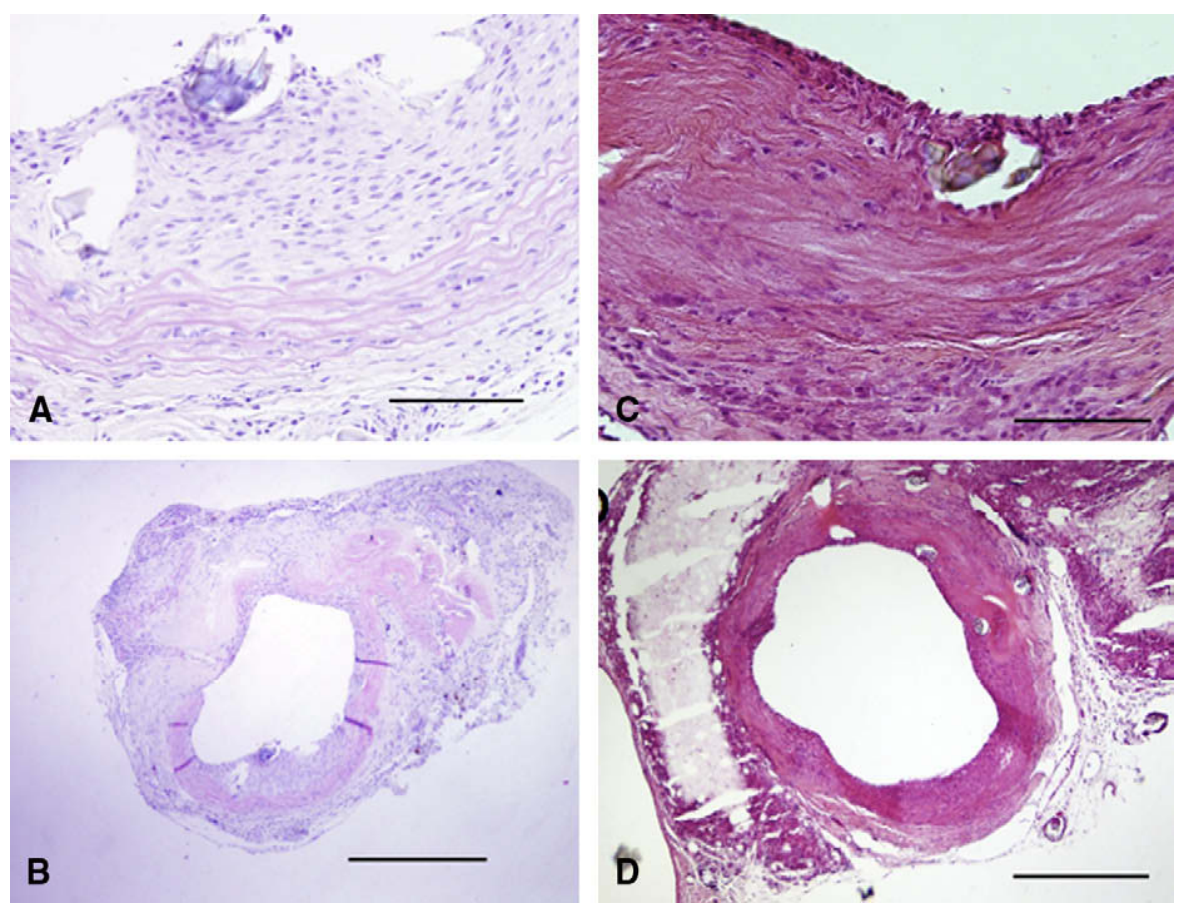

FIGURE 5. Histologic findings in the anastomotic site 2 weeks after operation. H\&E staining of control group $\times 200$ (A) and $\times 40$ (B), and TEBN containing $1.0 \mathrm{wt} \%$ tacrolimus $\times 200(\mathrm{C})$ and $\times 40$ (D). Neointimal hyperplasia is significantly decreased in TEBN containing $1.0 \mathrm{wt} \%$ tacrolimus. A and C, Scale bar $=$ $100 \mu \mathrm{m}$. B and D, Scale bar $=500 \mu \mathrm{m}$. 

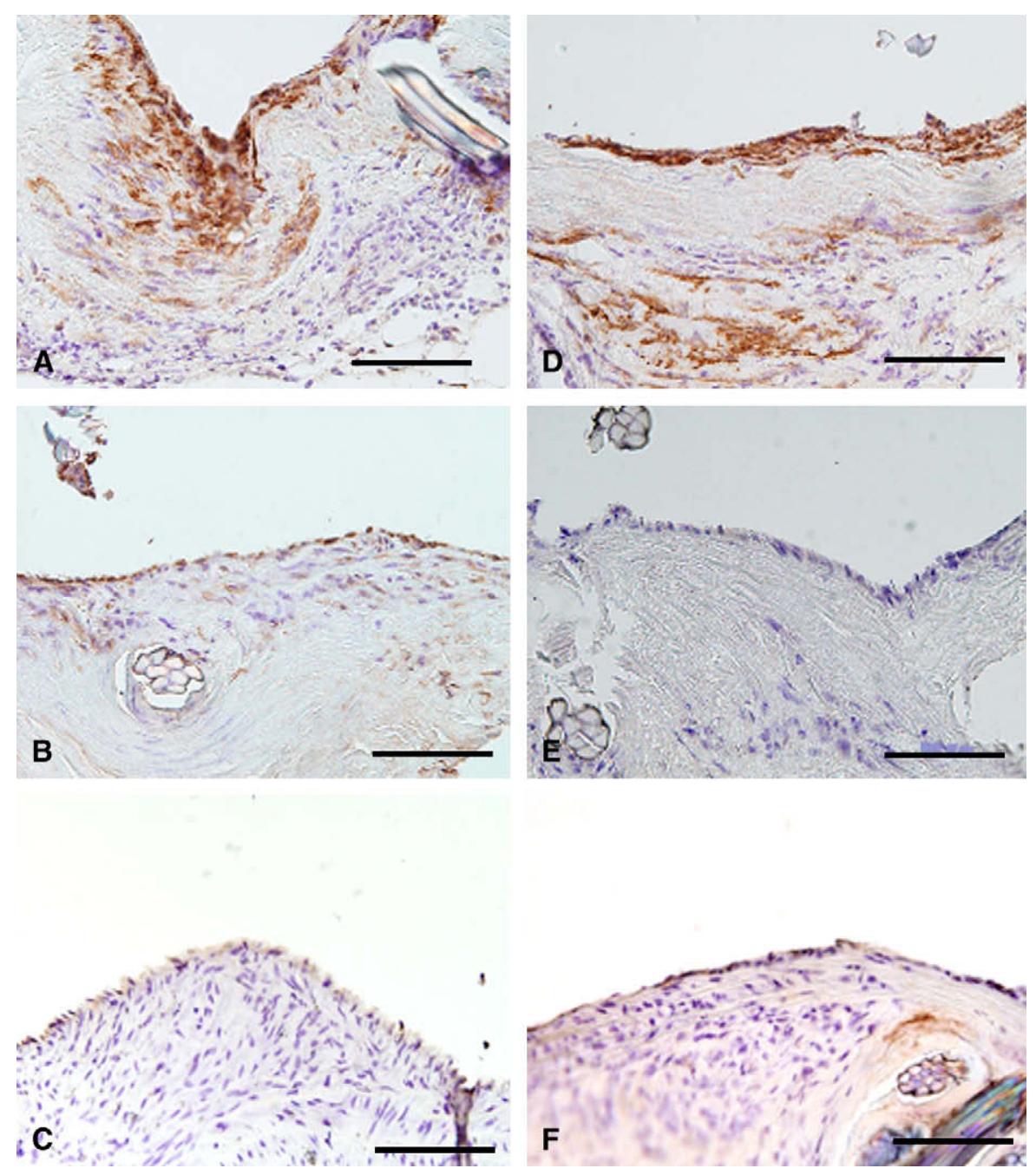

FIGURE 6. Immunohistochemical staining in the anastomotic site 2 weeks after operation. Micrographs (original magnification, $\times 200$ ) are representative photographs. A-C, Control group. D-F, TEBN-containing $1.0 \mathrm{wt} \%$ tacrolimus group. A and D, Stained with alpha-smooth muscle actin antibody. Alphasmooth muscle actin antibody-positive cells are shown in neointimal hyperplasia and adventitia. B and E, Stained with SMemb. SMemb-positive cells are shown in neointimal hyperplasia in control group. The longitudinal sections of (C) and (F) are stained with Factor VIII. Factor VIII-positive cells cover the surface of the inner lumen. Scale bar $=100 \mu \mathrm{m}$.

severity of allograft rejection after organ transplantation. It has also been used to treat other inflammatory conditions, such as atopic dermatitis. This agent has been shown to suppress T-cell activation and to inhibit the release of proinflammatory cytokines by binding to a cytosolic protein FKBP (FK506 binding protein). ${ }^{15}$ The drug FKBP complex stably associates with calcineurin and inhibits the serine/threonine phosphatase activity of this $\mathrm{Ca}^{2+}$-dependent enzyme, thus inhibiting calcineurin-dependent activation of lymphokine expression, apoptosis, and degranulation. Tacrolimus has been demonstrated to inhibit several steps of the cascade leading to neointimal proliferation. ${ }^{16}$ Inhibition of neointimal growth causing vascular smooth muscle cell (VSMC) proliferation was associated with reduced mitotic activity and diminished alpha-actin-positive smooth muscle cells in the intimal layer.
Sirolimus and tacrolimus are macrolide antibiotics that share potent immunosuppressive and anti-inflammatory properties. Both bind to the immunophilin FKBP but trigger different downstream mediators. Sirolimus inhibited VSMC proliferation and migration in vitro and decreased stent restenosis in experimental animals and human cases. ${ }^{17-20}$ External application of sirolimus by polymer sheets reportedly resulted in anastomotic stricture inhibition of neointimal hyperplasia. ${ }^{12}$ However, recent reports associated with sirolimus-eluting stents revealed subacute stent thrombosis and endothelial dysfunction, indicating insufficient reendothelialization by sirolimus. ${ }^{21,22}$ Matter and colleagues ${ }^{23}$ reported that tacrolimus was 100 times less potent than sirolimus for inhibiting VSMC proliferation or migration but exerted less antiproliferative effects on endothelial cells 
compared with sirolimus. They emphasized that sirolimus inhibited endothelial cell proliferation and migration, and induced late thrombosis and delayed endothelial recovery. Fukuda and colleagues ${ }^{24}$ found that potent inhibitory effects of sirolimus on vascular repair occur via stem and progenitor cells in vitro, and that perivascular application of the drug not only inhibited wire injury-induced neointima formation in mice but also retarded reendothelization. Thus, we chose tacrolimus (not sirolimus) in this study to avoid the abovementioned disadvantage of sirolimus.

Paclitaxel is also a powerful candidate as an antihyperplasia agent, similar to sirolimus. Paclitaxel-eluting coronary stents (Taxsus, Boston Scientific Corp, Natick, Mass) are commercially available worldwide. We examined the effect of paclitaxel in a pilot study with $1.0 \mathrm{wt} \%$ paclitaxel-eluting biodegradable nanofiber. The effects of paclitaxel on vascular tissue were stronger than those of tacrolimus. There was no endothelialization, and the number of cells was decreased in the intimal and medial layers (data not shown). Therefore, we did not apply paclitaxel in our experiments.

The preventative effect on neointimal hyperplasia from the adventitial side has been reported. ${ }^{11,12}$ The adventitia plays a great role in the maintenance of the integrity of the inner layers of the arterial wall. Injury to the adventitia, and more particularly to the vasa vasorum, can induce intimal lesion formation despite an intact endothelium. The adventitial myofibroblasts can migrate into the neointima and synthesize several components of the extracellular matrix. $^{25-27}$ An advantage of perivascular delivery is that the system is not in direct contact with blood and may reduce the risk of thrombosis, even in association with the reactively high dosage of the drug.

TEBN produced by electrospinning was assessed as a possible drug delivery device for this type of application. The shape of our new material, which consisted of nano-scale "cotton-wool" configuration fiber, has not been previously reported. Compared with gel and film and other types of polymer, our nano-scale "cotton-wool" configuration fiber has a distinct advantage for local application on any type of anastomotic suture site and for drug administration in a sustaining fashion. This nanofiber has been characterized with respect to the morphology, proinflammatory response drug distribution and release profiles, and ability of released tacrolimus to inhibit the proliferation of VSMC. Proliferation of VSMC is highest in the first week after injury and can continue 14 to 28 days depending on the severity of the injury. ${ }^{26}$ We observed anastomotic stricture in our experimental (rat abdominal aortic anastomosis) model during 1, 2, 3, 4, and 8 weeks (data not shown). The neointimal hyperplasia of the anastomosis was the worst at 2 weeks during the 8-week follow-up. Therefore, we decided to evaluate anastomotic stricture at 2 weeks after operation. Meanwhile, according to data of our in vivo release test, tacrolimus concentrations released from TEBN revealed a logarithmic curve showing a fast initial burst phase during the first few days followed by a slow sustained phase during the following 4 weeks. This releasing pattern may provide an appropriate time period for rat aortic vascular repair. If this is applied to a human anastomotic stricture, it should be optimized for humans in terms of the time of the stricture.

\section{Study Limitations}

This study was based on a rat aortic anastomosis model. The vascular healing process of the rat may be different from that of other animals and human beings. The tissue reaction of the rat aortic anastomosis was worst 2 weeks after operation and gradually regressed during 4 to 8 weeks.

Our anastomotic stricture model was established by end-to-end aortic anastomosis, preventing us from correctly evaluating other types of anastomosis (eg, end-to-side anastomosis, which is usually performed in coronary artery bypass grafting). End-to-end anastomosis has morphologic and hemodynamic differences from end-to-side arterial anastomosis. There may be a histologic difference between aortic anastomosis and peripheral arterial anastomosis. Thus, we could not correctly assess the peripheral arterial anastomotic stricture from our results.

Our method for measuring an intimal area was limited. We carefully avoided cutting oblique sections, but it is not altogether avoidable because of the short-axis section and paraffin-embedded fixed sample cut into a 5 -mm section. However, we believe that over- and underestimation of the intimal area can be prevented by taking the average of 3 sections.

The maximum tacrolimus content in the TEBN was 1.0 $\mathrm{wt} \%$ because of technical limitations. We could not test more than $1.0 \mathrm{wt} \%$ TEBN.

\section{CONCLUSIONS}

We developed a novel controlled drug-delivery device called "TEBN." This tacrolimus-releasing biodegradable nanofiber "cotton-wool"-like formation reduced neointimal hyperplasia and preserved endothelialization in a rat model, suggesting that it is a promising material for vascular anastomosis.

\section{References}

1. Ogus TN, Basaran M, Selimoglu O, Yildirim T, Ogus H, Ozcan H. Long-term results of the left anterior descending coronary artery reconstruction with left internal thoracic artery. Ann Thorac Surg. 2007;83:496-501.

2. Souza DS, Johansson B, Bojö L, Karlsson R, Geijer H, Filbey D, et al. Harvesting the saphenous vein with surrounding tissue for CABG provides long-term graft patency comparable to the left internal thoracic artery: results of a randomized longitudinal trial. J Thorac Cardiovasc Surg. 2007;132:373-8.

3. Mack MJ, Osborne JA, Shennib H. Arterial graft patency in coronary artery bypass grafting: what do we really know? Ann Thorac Surg. 1998;66:1055-9.

4. Roy-Chaudhury P, Kelly BS, Zhang J, Narayana A, Desai P, Melham M, et al. Hemodialysis vascular access dysfunction: from pathophysiology to novel therapies. Blood Purif. 2003;21:99-110.

5. Kapadia MR, Popowich DA, Kibbe MR. Modified prosthetic vascular conduits. Circulation. 2008;117:1873-82. 
6. Hosono M, Ueda M, Suehiro S, Suehiro S, Sasaki Y, Shibata T, et al. Neointimal formation at the sites of anastomosis of the internal thoracic artery grafts after coronary artery bypass grafting in human subjects: an immunohistochemical analysis. J Thorac Cardiovasc Surg. 2000;120:319-28.

7. Hannan EL, Wu C, Walford G, Culliford AT, Gold JP, Smith CR, et al. Drug-eluting stents vs. coronary-artery bypass grafting in multivessel coronary disease. N Engl J Med. 2008;358:331-41.

8. Moses JW, Leon MB, Popma JJ, Fitzgerald PJ, Holmes DR, O'Shaughnessy C, et al. Sirolimus-eluting stents versus standard stents in patients with stenosis in a native coronary artery. $N$ Engl J Med. 2003;349:1315-23.

9. Colombo A, Drzewiecki J, Banning A, Grube E, Hauptmann K, Silber S, et al. Randomized study to assess the effectiveness of slow- and moderate-release polymer-based paclitaxel-eluting stents for coronary artery lesions. Circulation. 2003; 108:788-94.

10. Bartorelli AL, Trabattoni D, Fabbiocchi F, Montorsi P, de Martini S, Calligaris G, et al. Synergy of passive coating and targeted drug delivery: the tacrolimus-eluting Janus CarboStent. J Interv Cardiol. 2003;16:499-505.

11. Signore PE, Machan LS, Jackson JK, Burt H, Bromley P, Wilson JE, et al. Complete inhibition of intimal hyperplasia by perivascular delivery of paclitaxel in balloon-injured rat carotid arteries. J Vasc Interv Radiol. 2001;12:79-88.

12. Kawatsu S, Oda K, Saiki Y, Tabata Y, Tabayashi K. External application of rapamycin-eluting film at anastomotic sites inhibits neointimal hyperplasia in a canine model. Ann Thorac Surg. 2007;84:560-7.

13. Kelly B, Melhem M, Zhang J, Kasting G, Li J, Krishnamoorthy M, et al. Perivascular paclitaxel wraps block arteriovenous graft stenosis in a pig model. Nephrol Dial Transplant. 2006;21:2425-31.

14. Schachner T, Zou Y, Oberhuber A, Tzankov A, Mairinger T, Laufer G, et al. Local application of rapamycin inhibits neointimal hyperplasia in experimental vein grafts. Ann Thorac Surg. 2004;77:1580-5.

15. Wallemacq PE, Reding R. FK506 (tacrolimus), a novel immunosuppressant in organ transplantation: clinical, biomedical, and analytical aspects. Clin Chem. 1993;39:2219-28.
16. Wiederrecht G, Lam E, Hung S, Martin M, Sigal N. The mechanism of action of FK-506 and cyclosporin A. Ann N Y Acad Sci. 1993;30:9-19.

17. Moses JW, Leon MB, Popma JJ, Fitzgerald PJ, Holmes DR, O'Shaughnessy C, et al. Sirolimus-eluting stents versus standard stents in patients with stenosis in a native coronary artery. $N$ Engl J Med. 2003;349:1315-23.

18. Marx SO, Jayaraman T, Go LO, Marks AR. Rapamycin-FKBP inhibits cell cycle regulators of proliferation in vascular smooth muscle cells. Circ Res. 1995;76:412-7.

19. Poon M, Marx SO, Gallo R, Badimon JJ, Taubman MB, Marks AR. Rapamycin inhibits vascular smooth muscle cell migration. J Clin Invest. 1996;98:2277-83.

20. Gallo R, Padurean A, Jayaraman T, Marx S, Roque M, Adelman S. Inhibition of intimal thickening after balloon angioplasty in porcine coronary arteries by targeting regulators of the cell cycle. Circulation. 1999;99:2164-70.

21. Finn AV, Joner M, Nakazawa G, Kolodgie F, Newell J, John MC, et al. Patholog ical correlates of late drug-eluting stent thrombosis strut coverage as a marker of endothelialization. Circulation. 2007;115:2435-41.

22. Hofma SH, van der Giessen WJ, van Dalen BM, Lemos PA, McFadden EP, Sianos G, et al. Indication of long-term endothelial dysfunction after sirolimuseluting stent implantation. Eur Heart J. 2006;27:166-70.

23. Matter CM, Rozenberg I, Jaschko A, Greutert H, Kurz DJ, Wnendt S, et al. Effects of tacrolimus or sirolimus on proliferation of vascular smooth muscle and endothelial cells. J Cardiovasc Pharmacol. 2006;48:286-92.

24. Fukuda D, Sata M, Tanaka K, Nagai R. Potent inhibitory effect of sirolimus on circulating vascular progenitor cells. Circulation. 2005;111:926-31.

25. Scott NA, Cipolla GD, Ross CE, Dunn B, Martin FH, Simonet L, et al. Identification of a potential role for the adventitia in vascular lesion formation after balloon overstretch injury of porcine coronary arteries. Circulation. 1996;93: 2178-87.

26. Shi Y, O'Brien JE Jr, Ala-Kokko L, Chung W, Mannion JD, Zalewski A. Origin of extracellular matrix synthesis during coronary repair. Circulation. 1997;95: 997-1006.

27. Shi Y, Pieniek M, Fard A, O'Brien J, Mannion JD, Zalewski A. Adventitial remodeling after coronary arterial injury. Circulation. 1996;93:340-8. 